\title{
Conociendo la fidelidad de las mujeres al examen de cáncer de cuello de útero
}

\author{
Recognizing the loyalty by women to cervical cancer
} screening

\section{Conhecendo a aderência das mulheres ao exame de câncer de colo de útero}

\author{
Pâmela Scarlatt Durães Oliveira ${ }^{1}$, Sérgio Vinicius Cardoso de Miranda ${ }^{2}$, \\ Gabriela Luize Guimarães Sanches ${ }^{3}$, Henrique Andrade Barbosa ${ }^{4}$ \\ ${ }^{1}$ Enfermeira. Mestranda em Ciências da Saúde (UNIMONTES). Especialista em Saúde da Família na \\ modalidade residência (UNIMONTES). Bolsista Capes \\ ${ }^{2}$ Enfermeiro. Doutorando em Ciências da Saúde, Universidade Estadual de Montes Claros/Unimontes, \\ Mestre em Saúde Pública, Fundação Oswaldo Cruz/FIOCRUZ-ENSP, Montes Claros (MG), Brasil \\ ${ }^{3}$ Enfermeira. Mestranda em Ciências da Saúde (UNIMONTES). Especialista em Trauma, Urgência e terapia \\ intensiva (FCMMG). Bolsista Fapemig \\ ${ }^{4}$ Enfermeiro. Mestre em Ciências da Saúde (UNIMONTES). Professor UNIMONTES, FASI \\ Cómo citar este artículo en edición digital: Oliveira, P.S.D., Miranda, S.V.C., Sanches, G.L.G. E \\ Barbosa, H.A. (2018). Conociendo la fidelidad de las mujeres al examen de cáncer de cuello de útero. \\ Cultura de los Cuidados (Edición digital), 22(52). Recuperado de \\ http://dx.doi.org/10.14198/cuid.2018.52.16 \\ Correspondência: Pâmela Scarlatt Durães Oliveira. Rua F, n. ${ }^{\circ} 43$, Bairro Vila Campos.CEP: 39.403-061 \\ - Montes Claros (MG), Brasil \\ Correo electrónico: pamela-scarlatt@bol.com.br \\ Recibido: 08/04/2018; Aceptado: 03/06/2018
}

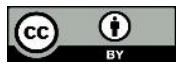

\section{ABSTRACT}

Introduction: Among the types of cancer, cervical cancer is one of the highest potential for prevention and cure.

Methods: The study is a community test that involves the intervention at the level of communities, instead of isolated individuals, being used to evaluate the efficacy and effectiveness of interventions.

Objective: The objective of this study is to relate the reasons for non-loyalty to preventive exam for cancer of the cervix and check the level of prior knowledge and later the lectures.

Results: Among the main reasons why women involved in this study reported not to perform the preventive exam regularly are the fear of examination, the shame of performing the examination and the lack of information.

Conclusion: Although there is some resistance in the carrying out of examinations for the prevention of cervicaluterine cancer, this being a great challenge for the teams of the Family Health Strategies.

Keywords: Women's Health, Health Education, Public Health, Primary Care, Cervical Cancer. 


\section{RESUMO}

Introdução: Dentre os tipos de câncer, o cervico-uterino é o que apresenta um dos mais elevados potenciais para prevenção e cura.

Métodos: $\mathrm{O}$ estudo trata-se de um ensaio comunitário que envolve a intervenção em nível de comunidades, ao invés de indivíduos isolados, sendo usado para avaliar a eficácia e efetividade de intervenções.

Objetivo: Este estudo tem como objetivo relacionar os motivos da não adesão ao exame preventivo de câncer de colo de útero e verificar o nível de conhecimento prévio e posterior às palestras.

Resultados: Dentre os principais motivos pelos quais as mulheres envolvidas nesse estudo relataram não realizar o exame preventivo regularmente estão o medo do exame, a vergonha de realizar o exame e o desconhecimento de informações.

Conclusão: Ainda percebe-se certa resistência na realização dos exames de prevenção do câncer cervico-uterino, sendo esse um grande desafio para as equipes de Estratégias de Saúde da Família.

Palavras chave: Saúde da mulher, Educação em saúde, Saúde Pública, Atenção Primária, Câncer de colo de útero.

\section{RESUMEN}

Introducción: Entre los tipos de cáncer, el cáncer cervical es uno del más alto potencial para la prevención y la curación.

Métodos: El estudio es una prueba comunitaria que involucra la intervención a nivel de las comunidades, en lugar de individuos aislados, siendo utilizado para evaluar la eficacia y la efectividad de las intervenciones.

Objetivo: El objetivo de este estudio es relacionar las razones para la no-fidelidad al examen preventivo para el cáncer de cuello uterino y comprobar el nivel de conocimiento previo y posterior a las conferencias.

Resultados: Entre las principales razones aportadas por las mujeres que participaron en este estudio, se destaca el miedo como causa principal de no realizar el examen preventivo regular. También la vergüenza de realizar el examen y, además, la falta de información.

Conclusión: Existe cierta resistencia en la realización de los exámenes para la prevención del cáncer cervico-uterino, siendo este un gran reto para los equipos de la Estrategia de Salud de la Familia.

Palabras clave: Salud de la mujer, Educación para la salud, Salud Pública, Atención Primaria, Cáncer de cuello uterino.

\section{INTRODUÇÃO}

O câncer cérvico-uterino (CCU) representa um sério problema de saúde pública, visto que são estimados para o ano de 2016 aproximadamente 16.340 mil novos casos, sendo a neoplasia de colo de útero umas das mais encontradas na população feminina, superada apenas pelo câncer de pele (não melanoma) e o câncer de mama (Brasil, 2014). Dentre os tipos de câncer, é o que apresenta um dos mais elevados potenciais para prevenção e cura podendo atingir até $100 \%$, desde que seja diagnosticado precocemente, e ainda pode ser tratado em nível ambulatorial em cerca de $80 \%$ dos casos (Casarin, Piccoli, 2011). Um dos fatores que interferem no tratamento é o fato de que algumas mulheres não realizam esse exame ou não retornam ao serviço de saúde para receber os resultados e, por conseguinte, estão expostos a doenças graves, sem tratamento (Junior, Oliveira, Sá, 2015).

Dentre os principais motivos para não 
realização do exame preventivo estão o desconhecimento da existência do câncer cérvico-uterino, da técnica e importância de realizar o exame, medo de se deparar com resultado positivo para câncer, sentimento de vergonha e constrangimento, questões culturais como a procura pelo setor hospitalar ao invés dos serviços de atenção primária, dificuldades de locomoção e acesso aos serviços de saúde, ou referentes às instituições como o horário de funcionamento (Silva et al., 2015).

Ao analisar a Atenção Primária à Saúde, cujo um dos objetivos é a promoção da saúde para a melhoria da qualidade de vida, pode-se perceber que os profissionais de saúde na Estratégia de Saúde da Família (ESF) têm grandes dificuldades em relação à adesão da população às reuniões de educação em saúde. Sabendo que os profissionais da enfermagem atuam diretamente neste âmbito, incorporando o papel de educador em saúde frente à assistência aos usuários, deve-se destacar a importância destas reuniões em transmitir conhecimento à população (Santos et al., 2015). É fundamental que os serviços de saúde orientem sobre o que é e qual a importância do exame preventivo, pois a sua realização periódica permite reduzir a mortalidade por câncer cérvico-uterino na população de risco, utilizando preferencialmente atividades de educação em saúde e estabelecimento de um vínculo entre paciente e profissional, firmado na confiança, respeito e visão do paciente como um ser holístico (Ferreira, 2009; Dias et al., 2015).

Sendo assim, traçaram-se os objetivos: caracterizar $o$ perfil das mulheres envolvidas no estudo, conhecer os motivos da não adesão ao exame preventivo de câncer de colo de útero e o nível de conhecimento prévio e posterior às palestras, conhecer a adesão das mulheres ao exame após participarem das palestras, conhecer o significado do exame preventivo do câncer de colo de útero, as experiências e as interações sociais na vida das mulheres participantes do estudo.

\section{MÉTODO}

$\mathrm{O}$ estudo trata-se de um ensaio comunitário que envolve a intervenção em nível de comunidades, ao invés de indivíduos isolados, sendo usado para avaliar a eficácia e efetividade de intervenções que busquem a prevenção primária através da modificação dos fatores de risco numa população. Foram feitas investigações de cunho quantitativo e qualitativo, sendo que nesse artigo são apresentados os dados referentes à parte qualitativa do estudo. Esse artigo também possui fundamentação teórica embasada no interacionismo simbólico que possibilita a compreensão do modo como os indivíduos interpretam os objetos e as suas interações com outras pessoas, valorizando assim o sentido que as coisas têm para o comportamento humano. Após essa análise das falas ocorreu o agrupamento das mesmas em forma de categorias com significados semelhantes (Dalfovo, Lana, Silveira, 2008; Carvalho, Borges, Rêgo, 2010).

Foi realizado um estudo longitudinal numa ESF de um município no norte de Minas Gerais, Brasil, com três abordagens realizadas pelos pesquisadores no grupo de mulheres. Os encontros ocorreram em forma de reuniões de educação em saúde com duração média de duas horas cada, e todas abordaram o tema do câncer de colo de útero e mama e cada uma, ainda, contou com temas de interesse para a população feminina para despertar a vontade de retornar às demais reuniões. O intervalo 
entre os grupos foi de aproximadamente sete dias, entre cada intervenção. Para a coleta de dados sociodemográficos foi utilizado um questionário com perguntas elaboradas com base na ficha de requisição de exame citopatológico do colo do útero normatizada pelo Ministério da Saúde e o formulário de consulta de enfermagem da ESF.

A amostra selecionada foi composta de 40 mulheres que não realizam o exame preventivo do câncer do colo de útero, regularmente, e 20 mulheres que realizam o exame, com idades entre 25 e 64 anos, sendo que tal recomendação apoiou-se em estudo realizado pela International Agency for Research on Cancer (IARC), publicado em 1986, e que envolveu oito países. Esse estudo, que serviu de base para a normatização vigente no mundo, sendo que essa nova faixa etária também é determinada pelo Instituto Nacional de Câncer (IARC, 1986). Tendo em vista a não adesão às reuniões, foi realizada busca ativa as participantes para comparecerem e as informações das palestras foram passadas pelas pesquisadoras na própria residência das participantes do estudo. As entrevistas também aconteceram na casa das mulheres, posteriormente ás reuniões e ao dia da busca ativa, em horário agendado. Essa segunda etapa foi realizada somente com aquelas participantes que se mostraram resistentes a marcação do exame de prevenção, mesmo após a intervenção da Educação em Saúde, realizando um atendimento mais personalizado a cada mulher.

Por se tratar de pesquisa envolvendo seres humanos, cumpriram-se os requisitos exigidos pela Resolução n. o $^{466 / 2012}$ do Conselho Nacional de Saúde, submetendo o projeto que originou esse estudo à apreciação do Comitê de Ética em Pesquisa da SOEBRAS com o parecer de aprovação n. ${ }^{\circ} 01627 / 11$.

\section{RESULTADOS}

No primeiro momento, buscou-se caracterizar o perfil sócio demográfico (idade, escolaridade, estado civil e renda mensal) da amostra de mulheres que participaram do estudo através de um questionário com perguntas abertas preenchido pelas mesmas. Por meio dos dados coletados, podemos verificar que a maioria das mulheres tem menos de 50 anos de idade, sendo que varia entre 25 a 64 anos. Quanto à ocupação, a maioria das mulheres respondeu que são autônomas e donas-decasa. Foi verificada também a renda familiar entre as mulheres e foi revelado que grande parte possui até 1,5 salários mínimos. Com base nas fichas preenchidas, os dados obtidos revelaram os fatores que dificultam o acesso ao exame preventivo de câncer de colo de útero, sendo que $25 \%$ das mulheres afirmaram ter medo de fazer o exame, 35\% relataram ter vergonha de fazer o exame e $40 \%$ revelaram trabalhar durante o período de funcionamento da ESF em que foi realizado o estudo.

\section{Categoria 1: O significado do exame preventivo do câncer de colo de útero.}

Sobre os aspectos sociais e individuais das mulheres relacionados a não realização periódica do exame Papanicolaou, acreditase que várias ideias e pensamentos culturalmente aceitos permeiam as comunidades locais e refletem os motivos pelos quais estas participantes não realizavam o exame cérvico-uterino periodicamente. Nas categorias seguintes são apresentados os principais motivos pelos quais as mulheres envolvidas nesse estudo relataram não realizar o exame preventivo regularmente. 


\section{Subcategoria 1 A: "medo de descobrir coisa ruim"}

Grande parte das mulheres entrevistadas relatou não realizar o exame preventivo por medo do procedimento ou de descobrir que possui o câncer.

Eu tenho medo do exame por que todo mundo que fez falou que doeu muito" (Margarida).

Eu tenho medo de fazer esse exame e descobrir que eu tenho alguma coisa ruim, prefiro morrer sem saber " (Rosa).

Eu tenho medo do exame por que nunca fiz, $e$ todo mundo que descobre essa doença morre, vai que eu faço o exame e descubro" (Girassol).

Quando eu vou no posto de saúde consultar com o médico e tem outras mulheres esperando pra fazer esse exame, elas sempre me falam que dá um desconforto e uma dorzinha, mais que elas fazem sempre, mais eu fico com medo mesmo assim neh (...)"(Violeta).

\section{Subcategoria 1 B: "a vergonha é maior que a necessidade de fazer esse exame"}

Outra razão apresentada pelas mulheres para a não-adesão ao exame preventivo foi a vergonha de expor seus corpos aos profissionais de saúde.

Eu não tenho coragem de ficar deixando qualquer um ver minhas partes não, pode não, só meu marido que pode ver, e a vergonha como fica? (Jasmim).

Eu fui criada pra ter vergonha, ninguém pode me ver sem roupa não (Cravo).

Eu tenho vergonha de me expor pro enfermeiro, ainda mais que ele é um homem! (...) A vergonha é maior que a necessidade de fazer esse exame (Lírio).

Eu fico meio assim da pessoa ver minhas intimidades, como que a enfermeira vai olhar pra minha cara depois de mer ver pelada? (Orquídea).

Guardar minha intimidade é mais importante que fazer esse exame" (Petúnia).
Subcategoria 1 C: "pensei que era só umas mulheres que precisavam fazer"

Em consonância com as informações encontradas na literatura, muitas mulheres não realizam o exame por que não tem conhecimento suficiente sobre o mesmo. As falas das entrevistadas evidenciam que a população carece de informações simples sobre a realização do exame de prevenção, como a idade de início de realização.

Uai, eu nem sabia que ainda tinha idade pra fazer esse exame, pensava que era só as menina nova que fica com um tanto de homem, vixi tem que fazer então (Alfazema).

Hum... Eu não sabia que as mulheres que não faz mais esses trem ainda tinha que fazer esse exame, eu pensei que nós que não tem marido estava livre disso (Anis).

Mulher nova igual eu já pode fazer esse exame? Mesmo se for solteira? (Amor-perfeito).

Me explica aí como que esse exame é feito, porque aí eu posso fazer, vou ficar menos cismada com ele (Flor-de-laranjeira).

Eu nunca fiz esse exame, pensei que era só umas mulheres que precisavam fazer, as que o médico indica por que tá com algum problema nas partes intimas (Glicínia).

Eu não sabia que no PSF fazia esse exame e que era tão simples assim, pensei que era uma coisa de outro mundo, terrível (Helicônia).

\section{Categoria 2: A experiência quanto ao exame de prevenção do câncer de colo de útero.}

Nessa categoria são destacadas as experiências negativas em relação ao exame e que fizeram com que as mulheres se tornassem resistentes á realização do mesmo.

\section{Subcategoria 2 A: "a experiência foi tão horrível que eu resolvi parar"}

Eu já fiz esse exame mais não voltei nem pra pegar o resultado porque até me esqueci (Azaléa). 
Eu trabalho em casa o dia todo, não tenho tempo pra ir repetir o exame e, além disso, eu não faço porque já fiz uns anos atrás e a experiência foi tão horrível que eu resolvi parar (Crisântemo).

Eu já tentei fazer o exame muitas vezes mais eu sou mãe e dona-de-casa, não tenho onde deixar meus filhos pra ir fazer o exame (Hortência).

Já marcaram esse exame pra mim uma vez, mais quando eu cheguei pra fazer me avisavam que a pessoa que faz os exames faltou no dia, ai eu desisti porque o PSF é muito longe pra eu ficar indo e voltando sempre (Flor-de-lis).

Eu já tentei fazer o exame, mais eu não tenho o cartão do SUS e eu já tentei fazer aí no PSF mais não consegui, então acho que esse exame não é importante assim não, porque se fosse eu teria conseguido marcar rapidinho (Copo-de-leite).

Eu só não faço o exame por que trabalho o dia todo (Cacto).

\section{Categoria 3: Interações sociais relacionadas ao exame de prevenção do câncer de colo de útero.}

\section{Subcategoria 3 A: "se dessa vez ele deixa eu fazer!"}

Além do medo, da vergonha e de experiências passadas ruins relacionadas à realização do exame de papanicolau existem fatores ligados aos relacionamentos e o meio social em que as mulheres convivem e que também contribuem para essa resistência na realização do exame.

Eu não gosto muito de comentar não, mais meu marido fica meio bravo quando eu falo de fazer esse exame, é melhor não caçar briga dentro de casa (Lavanda).

Minha irmã fez esse exame e descobriu o câncer, não demorou tempo nenhum e ela morreu, e eu sei que foi por que descobriu, se não tivesse descobrido tava viva até hoje aqui com nós (Flor-do-campo).

Eu não faço esse exame não porque eu nem sei quem é a enfermeira aí desse PSF, ela conhece nós da população, eu só faço o exame se for com alguém que eu conheço (Flor-de-lótus).

Minha vizinha aqui do lado falou que passou foi mal quando fez esse exame por que doeu demais e já me avisou pra eu não mexer com isso não que eu também vou é ficar doente de tanta dor (Margarida).

Ninguém da minha família faz esse exame depois que minha mãe morreu de câncer no útero, prefiro ficar sem saber que eu tenho alguma coisa ruim, essas doenças assim não tem cura não e você morre mais rápido quando descobre (Rosa).

Eu e minha amiga combinamos que o dia que uma tomar coragem a outra também toma e nós vamos juntas fazer esse exame. Ainda bem que esse dia nunca chega, não se depender de mim depois de tanta coisa ruim que as pessoas descobrem consultando, eu nem em médico vou, de tipo nenhum (Tulipa).

Você pode até marcar o exame pra mim, mais eu vou perguntar meu namorado se dessa vez ele deixa eu ir lá fazer (Flor de Iris).

\section{DISCUSSÃO}

Os resultados revelam que a maioria das participantes estava na faixa etária de até 50 anos, período de vida que constitui fator de risco para desenvolvimento de câncer de colo uterino, cuja idade exige que se seja intensificada as medidas de prevenção. Este valor coincide com o estudo de Gerónimo et al. (2011). A maioria das mulheres também responderam ser autônomas e donas-decasa, dado que coincide com o estudo de Brischiliari. A maioria das mulheres apresenta um baixo nível socioeconômico, sendo este um dos fatores de risco para desenvolvimento do câncer de colo de útero, além da multiplicidade de parceiros, tabagismo, infecção por HPV, tabagismo e início precoce de atividade sexual. Evidenciou-se a necessidade da Educação 
em Saúde voltada à sexualidade, pois todas as participantes relataram que já tiveram relações sem preservativos. Segundo a literatura, este é um dos fatores de rico para o câncer cervical (Brischiliari, 2012).

É de fundamental importância que os serviços de saúde também orientem sobre o que é, e qual a importância do exame preventivo, pois a sua realização periódica pelas mulheres com idade entre 25 e 64 anos (nova faixa etária determinada pelo Ministério da Saúde para realização do exame) permite reduzir a mortalidade por CCU na população de risco, utilizando preferencialmente atividades de educação em saúde e estabelecimento de um vínculo entre cliente e profissional, firmado na confiança, respeito e visão do paciente como um ser holístico (Ferreira, 2009).

Para que isso aconteça é necessário que os enfermeiros conheçam os problemas enfrentados por sua população em se tratando de razões para não-adesão ao exame preventivo. Dentre os motivos pelos quais as mulheres envolvidas nesse estudo relataram não realizar o exame preventivo de câncer de colo de útero regularmente estão o medo exame, a vergonha de realizar o exame, o desconhecimento de informações sobre o exame, fatores externos á vontade das mulheres, experiências passadas ruins e a grande maioria relatou não realizar o exame porque trabalha durante o período de funcionamento da unidade de saúde, ou seja, por dificuldade de acesso aos serviços de saúde.

Referente à categoria 1 , subcategoria do medo do exame, um dos fatores que impedem o acesso ao exame merece um pouco mais de reflexão. $\mathrm{O}$ diagnóstico do câncer possui um efeito devastador sobre a usuária, trazendo a idéia de aproximação com a morte, possíveis mutilações e a dor proveniente dos tratamentos, trazendo grandes impactos na vida da mulher e da sua família.

Muitas mulheres não realizavam o exame preventivo com medo de se deparar com um resultado positivo para o câncer cérvicouterino. A realização do exame preventivo torna-se assim provocador de tensões emocionais que precisam ser mais bem trabalhadas. Vários estudos relatam que muitas vezes entre os pacientes, existe o conceito de câncer de forma mistificada, visto como doença fatal, o que desvaloriza a prevenção por meio de uma postura conformista de ter uma doença contra a qual nada se pode fazer (Silva et al., 2015; Burille, Schwartz, Zillmer, 2013).

Vale ressaltar ainda que muitas mulheres que não realizam o exame e percebem o mesmo como um processo agressivo, físico e que as afetava emocionalmente, contudo com a oportunidade de se discutir acerca do exame, esses sentimentos poderiam ser amenizados, demonstrando mais uma vez a importância de que a informação seja transmitida as pacientes através de da Educação em Saúde (Silva et al., 2013).

Para a subcategoria que relaciona a vergonha como barreira para a realização do exame, o presente estudo revelou ainda que muitas mulheres não realizem o exame por vergonha do procedimento que expõe muito os seus corpos para profissionais de saúde desconhecidos, o que está em conformidade com o que foi dito em outras pesquisas. Porém, mesmo consciente da necessidade de fazê-lo, a vergonha ainda afasta a mulher do serviço de saúde (Silva Souza et al., 2013).

Neste caso, é necessário que haja uma maior sensibilidade e compreensão por parte dos profissionais durante a realização do exame. O sentimento de vergonha exacerbado dificulta a realização do exame, pois a mulher não consegue relaxar, tornando, consequentemente, o exame mais 
doloroso e ocasionando contrações da musculatura pélvica (Moura, Silva, Farias, Feitoza, 2012).

Quanto a falta de conhecimento sobre o exame, a importância da informação no processo de realização do exame preventivo demonstrou-se a partir da constatação de que grande parte das mulheres que não se submeteu ao exame preventivo não o fez por desconhecimento da necessidade do mesmo e dos aspectos ligados à doença. Muitas vezes a falta de conhecimento sobre o exame está relacionado a baixa escolaridade, sendo fator determinante na omissão de mulheres na realização do exame preventivo (Carvalho et al., 2016).

Vale ressaltar ainda que dentre as mulheres também não há um conhecimento científico sobre o câncer de colo de útero e as ideias estão focadas em conhecimentos populares. Isso pode ocorrer devido à falta de orientações teóricas repassadas pelos profissionais da unidade de saúde, deixando claro mais uma vez que os profissionais da área devem promover a educação em saúde e não somente esperar que as usuárias busquem uma consulta, mas que usem da criatividade, através de campanhas educativas, visita às escolas dentre outras ações (Silva Sousa, et al., 2016).

Muitas mulheres não realizam o exame de prevenção devido as experiências prévias ruins em relação ao exame como as barreiras relativas aos serviços de saúde, como o horário de trabalho que é incompatível com as jornadas de serviço das mulheres ou até mesmo a distância do serviço de saúde da casa dessas mulheres (Moura, Silva, Farias, Feitoza, 2012).

Um estudo multicêntrico internacional coordenado pela Organização Mundial de Saúde (OMS) e realizado em oito países (no Brasil, em São Paulo - 2003), evidenciou-se que $11,1 \%$ das entrevistadas tinham dificuldades para marcar o exame/não havia vaga e $6 \%$ relataram falta de tempo para realizá-lo. Na literatura, são escassos os estudos que relatam os motivos relacionados às unidades de saúde como dificultadores para a realização do exame de Papanicolau (Silva, Silveira, Gregório, 2012).

Outros fatores que influenciam a nãorealização do exame preventivo estão associados ao papel da mulher no cuidado com a casa e os filhos, relacionados ao dia-adia, repleto de afazeres que socialmente se veem como necessários, considerando as funções das mulheres que se somam às atividades de casa e ao papel de mãe e à condição de trabalhador fora de casa ${ }^{4}$. Outros motivos como o fato de se esquecer de buscar os resultados também estão em consonância com o que foi observado nos estudos (Aguilar, Soares, 2015).

$\mathrm{Na}$ categoria relacionada às interações sociais relacionadas ao exame de prevenção do câncer de colo de útero foi observado que muitas mulheres relataram não realizar o exame preventivo por falta de um relacionamento com o enfermeiro ou médico que trabalha no ESF do seu bairro. É de vital importância a formação de vínculo entre a enfermeira e a cliente, que pode ser percebido na fala de algumas mulheres, demonstrando claramente o teor ético que essa atuação possui. Cabe a enfermeira elucidar à mulher que todas as informações derivadas da consulta serão mantidas em sigilo. Ao resguardar a identidade da mulher, o profissional inicia um vínculo que pode perdurar nas ações de saúde a esse indivíduo (Silva et al., 2013).

Quando se discute sobre o câncer, a maioria dos casos citados são os ruins, isto é, aqueles em que a cura não foi possível e a morte foi o desfecho, depois de muita dor e sofrimento. Os profissionais de saúde devem sempre lembrar às pessoas e dar 
exemplos dos casos que realmente deram certo, em que os pacientes conseguiram a cura e continuam seguindo suas vidas normalmente. Assim como no estudo de Ferreira muitas mulheres não realizam o exame por medo de se deparar com um resultado positivo, como aconteceu com algum ente querido ou algum conhecido que teve um final trágico após o diagnóstico (Silva et al., 2015).

Apesar de ser a prevenção a forma mais eficaz para evitar o câncer, a adesão dos indivíduos aos comportamentos preventivos de saúde ainda é dificultada devido aos modelos e padrões de reconhecimento e valorização de aspectos culturais que não contribuem na mudança de atitudes, e em conformidade com o estudo de Ferreira muitas mulheres não realizam o exame por conta de costumes familiares passados para as mesmas ou até mesmo da opinião e de experiências de relacionamentos que compõe o ciclo social dessa mulher, assim como no estudo de Silva que os maridos também interferiam negativamente na realização do exame (Silva et al., 2015; Silva et al., 2013).

\section{Limitações do estudo e sugestões para futuras investigações}

Dentre as principais dificuldades encontradas para a realização desse estudo pode ser citado a baixa adesão das mulheres às atividades educativas promovidas na ESF, sendo que os motivos para essa não adesão devem ser melhor investigados, visto que esse é um problema que acomete todas as faixas etárias das populações assistidas pela atenção primária.

Vale ressaltar que a integração do sistema de saúde com a transmissão da informação é a principal forma de influência sobre a mudança de comportamento das pessoas, desde que ele respeite os valores e as crenças da população, e que não realize a ação de informação somente baseada no seu conhecimento, mas que crie um espaço de aprendizado mútuo com a clientela, sendo, portanto de vital importância para aumentar a adesão ás boas práticas de saúde, incluindo o exame preventivo (Silva Souza et al., 2013).

Um ponto importante a ser ressaltado são as dificuldades não na realização do estudo, mais aquelas que estão relacionadas ao processo de trabalho do serviço de saúde, que apesar de se prontificar para marcar os exames para as mulheres em atraso com o exame envolvidas no presente estudo, acabou desmarcando todos os exames sem aviso prévio e até a conclusão desse estudo os exames ainda não haviam sido marcados. $\mathrm{O}$ horário de funcionamento do serviço também é incompatível com os horários de trabalho das mulheres, visto que muitas não puderam realizar o exame por esse motivo, sendo assim é viável que sejam estudadas novas propostas mais flexíveis de horários de funcionamento das ESF's e que se adequem melhor a vida de sua população.

Propõe-se ainda que sejam estudadas maneiras mais eficazes de captação das mulheres para a realização do exame preventivo, visto que através desse estudo foi observado que os motivos para a não adesão vão além da falta de informação, envolvendo questões culturais mais profundas que estão fundamentadas em crenças, mitos e tabus, que têm um grande significado para o indivíduo. Esses valores se não forem associados à realidade podem representar uma grande barreira para os profissionais que atuam na promoção, reabilitação da saúde e prevenção de doença. 


\section{CONSIDERAÇÕES FINAIS}

Apesar da existência de políticas públicas voltadas exclusivamente para a população feminina do Brasil, ainda percebe-se certa resistência na realização dos exames de prevenção do câncer cérvico-uterino, sendo esse um grande desafio para as equipes de ESF's, que devem procurar conhecer as particularidades de sua população e as razões para a não-adesão ao exame.

Discutindo-se sobre esses motivos é necessário que existam campanhas voltadas para as mulheres das microáreas, com a busca ativa por meio de visitas regulares dos agentes comunitários de saúde, reuniões na comunidade pelas equipes saúde da família e ações educativas. A melhoria da qualidade do serviço, como a diminuição do tempo de espera para atendimento, horários diferenciados para as trabalhadoras e respeito à privacidade são outras estratégias que devem ser utilizadas no sentido de captar esta população para a prevenção do câncer cérvico-uterino.

Conflitos de interesse: Os autores declaram não existirem conflitos de interesses.

\section{REFERÊNCIAS}

\footnotetext{
- Aguilar, R.P. \& Soares, D.A. (2015). Barreiras à realização do exame Papanicolau: perspectivas de usuárias e profissionais da Estratégia de Saúde da Família da cidade de Vitória da Conquista-BA. Physis: Revista de Saúde Coletiva, 25(2), 359-379.

- Brasil. (2014). Instituto Nacional do Câncer (INCA). Dados sobre câncer de colo do útero. Brasilia: Ministério da Saúde (MS). Recuperado de http://www.inca.org.br.

- Brischiliari, S.C.R., et al. (2012). Papanicolaou na pós-menopausa: fatores associados a sua não realização. Caderno de Saúde Pública, 28(10), 19761984.

- Burille, A., Schwartz, E. \& Zillmer, J.G.V. (2013). Changes in the daily life of men with cancer: introducing one of the interfaces of getting sick.
}

Revista de Pesquisa: Cuidado é Fundamental Online, 5(2), 3539-3548.

- Carvalho, V.D., Borges, L.O. \& Rêgo, D.P. (2010). Interacionismo Simbólico: Origens, Pressupostos e Contribuições aos Estudos em Psicologia Social. Revista Psicologia, ciência e profissão, 30(1):146-161.

- Carvalho, V.F., et al. (2016). Acesso ao exame papanicolau por usuárias do Sistema Único de Saúde. Northeast Network Nursing Journal, 17(2), 2225

- Casarin, M.R. \& Piccoli, J.C.E. (2011). Educação em Saúde para Prevenção do Câncer de Colo do Útero em Mulheres do Município de Santo Ângelo/RS. Ciência \& Saúde Coletiva, 16(9), 3925-3932.

- Dalfovo, M.S., Lana, R.A. \& Silveira, A. (2008). Métodos quantitativos e qualitativos: um resgate teórico. Revista Interdisciplinar Científica Aplicada, 2(4):01-13.

- Dias, E.G., et al. (2015). Avaliação do conhecimento em relação à prevenção do câncer do colo uterino entre mulheres de uma Unidade de Saúde. Revista de Epidemiologia e Controle de Infecção, 5(3)15-22

- Ferreira, M.L.S.M. (2009). Motivos que influenciam a não-realização do exame de papanicolaou segundo a percepção de mulheres. Escola Anna Nery Revista Enfermagem, 213(2), 378-38.

- Gerónimo, N., et al. (2011). Prevalência de cáncer de cuello uterino y cáncer de seno en Yopa 1, Casanare, Colombia. Investigaciones Andina, 13(22), 162-173.

- Iarc working group on evaluation of cervical cancer screening programmes. (1986). Cervical Cancer Screening Programmes. Screening for squamous cervical cancer: duration of low risk after negative results of cervical cytology and its implication for screening policies. 293, 659-664.

- Junior, J.C.O., de Oliveira, L.D. \& Sá, R.M. (2015). Fatores de adesão e não adesão das mulheres ao exame colpacitológico. Gestão e Saúde, 6(1), 184-200. - Moura, A.D.A., Silva, S.M.G., Farias, L.M. \& Feitoza, A.R. (2012). Conhecimento e motivações das mulheres acerca do exame de papanicolaou: subsídios para a prática de enfermagem. Rev. Rene. Fortaleza, 11(01), 94-104.

- Santos, A.M., et al. (2015). Desafios à gestão do trabalho e educação permanente em saúde para a produção do cuidado na estratégia saúde da família. Revista de APS, 18(1),335-342.

- Silva, E.C.A., et al. (2015). Conhecimento das mulheres de 18 a 50 anos de idade sobre a importância do exame de papanicolaou na prevenção do câncer de colo uterino no município de Turvânia-GO. Revista Eletrônica Faculdade Montes Belos, 8(4).

- Silva, S.E.D., et al. (2013). As representações sociais do câncer de mama e no colo do útero no 
conhecimento da enfermagem brasileira. Gestão $e$ Saúde, 4(3), 1130-1145.

- Silva Sousa, I.G., et al. (2016). Prevenção do câncer de colo uterino: percepções de mulheres ao primeiro exame e atitudes profissionais. Northeast Network Nursing Journal, 9(2), 332-335.

- Silva Souza, G.D., et al. (2013). A concepção das mulheres de Mirandópolis-São Paulo acerca do exame de papanicolau. Revista de Enfermagem da
UFSM, 3(3), 470-479.

- Silva, S.R., Silveira, C.F. \& Gregório, C.C.M. (2012). Motivos alegados para a não realização do exame de papanicolaou, segundo mulheres em tratamento quimioterápico contra o câncer do colo uterino. Revista Mineira de Enfermagem, 16(4), 579-587. 